\title{
Cavity Enhanced Reflector Based Hybrid Silicon Laser
}

\author{
Y. De Koninck, G. Roelkens and R. Baets \\ Department of Information Technology, Photonics Research Group, Ghent University - IMEC \\ Sint-Pietersnieuwstraat 41 B-9000 Ghent, Belgium \\ email: yannick.dekoninck@intec.ugent.be
}

\begin{abstract}
We present a novel approach to integrate III-V based hybrid lasers on a silicon platform using resonant grating structures as compact and highly-reflective mirrors.

Index Terms-Silicon Photonics, Heterogeneous Integration, Hybrid Lasers, Grating Reflector, Grating Resonator
\end{abstract}

\section{INTRODUCTION}

In recent years, Silicon-On-Insulator (SOI) has gained significant momentum as a novel platform for integrated optical circuits. Its large refractive index contrast allows for ultracompact waveguide circuits and due to its compatibility with mainstream CMOS fabrication facilities, cheap production and seamless integration of photonic and electronic functionality on the same chip will become available. This, on its turn, enables exciting new applications such as ultra-fast optical communication links between different chips on a circuit board or low-cost lab-on-a-chip solutions. Most optical components necessary to build these systems, such as photonic wires, filters, couplers and splitters, are readily available. But another crucial building block, an on-chip laser source, has been posing a problem for a long time.

Due to its indirect bandgap, light emission is extremely inefficient in silicon, making it unsuitable as a gain material. One of the solutions proposed to tackle this issue is to bond a direct-bandgap active material, preferably a III-V semiconductor compound, on top of the SOI substrate and pattern both the silicon and the active layer such that a laser cavity arises. This principle has proven to work in several different configurations, such as for example grating based lasers [1] [2] and a microdisk laser [3].

In this article we propose a promising new approach to obtain a compact, efficient and single mode hybrid silicon laser based on cavity enhanced reflectors (CER).

\section{EFFICIENT HYBRID LASER CAVITY DESIGN}

As stated before, the hybrid platform consists of an SOI substrate with a slab of III-V material bonded on top and a thin layer of oxide or polymer in between. In a crosssectional cut we find two waveguides, one in the silicon and one in the active layer as shown in figure 1. Inside the cavity the optical mode should be confined primarily to the III-V waveguide to maximize the overlap with the active quantum wells, optimizing power amplification.

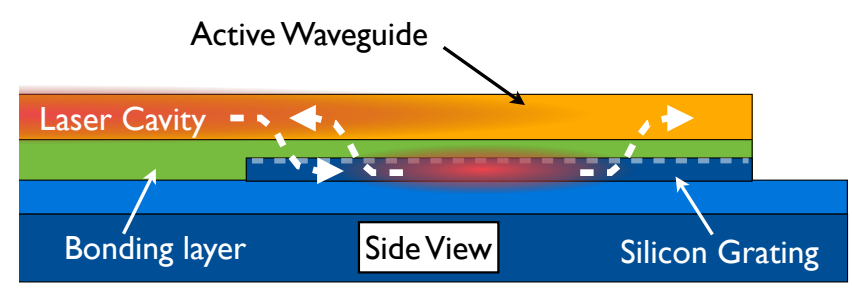

Fig. 1. Schematic view of a resonant cavity reflector in a hybrid silicon laser.

At the edges of the gain section, mirrors make sure that the optical power is reflected back into the cavity. Bragg gratings are widely used for this purpose for their high reflectivity and precise control of reflectivity spectrum during fabrication. To limit the number of process steps on the III-V layer, this grating is preferably patterned in the silicon waveguide. If operated at the Bragg wavelength, the silicon grating can be treated as a small periodic perturbation to the active waveguide, evanescently reflecting the forward propagating wave into a backward propagating wave. Unfortunately, the silicon and active waveguide are usually spaced too far apart to result in significant reflection over a short distance, forcing us to use long grating reflectors.

\section{CAVity Enhanced Reflectors}

It is possible though to obtain high reflection using short gratings $(\sim 20 \mu \mathrm{m})$ by making only a minor adjustment to the grating. Due to their proximity, there is, albeit limited, coupling between the active and the silicon waveguide, so light will leak from the first into the latter. At first sight this limited fraction can be neglected, unless the grating forms a resonant cavity, which can be done by adding a phase shifting section of half a period at the center of the grating. In this case, power will build up inside the grating cavity and eventually couple back into the active waveguide. If both waveguides are phase-matched and critically coupled, the light that couples back co-directionally to the incoming signal will interfere destructively with the latter, resulting in zero net transmission. The counter-directional light will reflect back into the laser cavity, providing the feedback required for laser operation. This process is also illustrated in figure 1. Note that this approach does not require ultra-thin $(<100 \mathrm{~nm})$ 
bonding layers as the exact coupling strength is not important as long as the losses in the cavity are sufficiently low.

\section{Simulation Results}

We have simulated the use of cavity enhanced reflectors extensively in the context of hybrid silicon lasers. To obtain reliable results we used the open-source eigenmode solver CAMFR [4] to calculate the first-order to first-order mode reflection of the active waveguide as a function of wavelength. All simulations were performed for a 60 period shallow etched grating $(70 \mathrm{~nm}$ etch depth, $300 \mathrm{~nm}$ period and $50 \%$ duty cycle) in a $220 \mathrm{~nm}$ high silicon wire. In our case the grating's stopband ranges from $1.54 \mu \mathrm{m}$ to $1.64 \mu \mathrm{m}$ with a Bragg wavelength of $1.59 \mu \mathrm{m}$. A $250 \mathrm{~nm}$ high InP active waveguide is bonded on top of the grating using a $350 \mathrm{~nm}$ thick BCB layer. The thickness of the active layer is chosen such that it is phase-matched to the silicon grating at the Bragg wavelength. In the case of the resonant cavity, the phase section is not exactly at the center of the grating, but shifted 4 periods to the left to counteract the asymmetric injection. This optimization increases the maximum reflection.

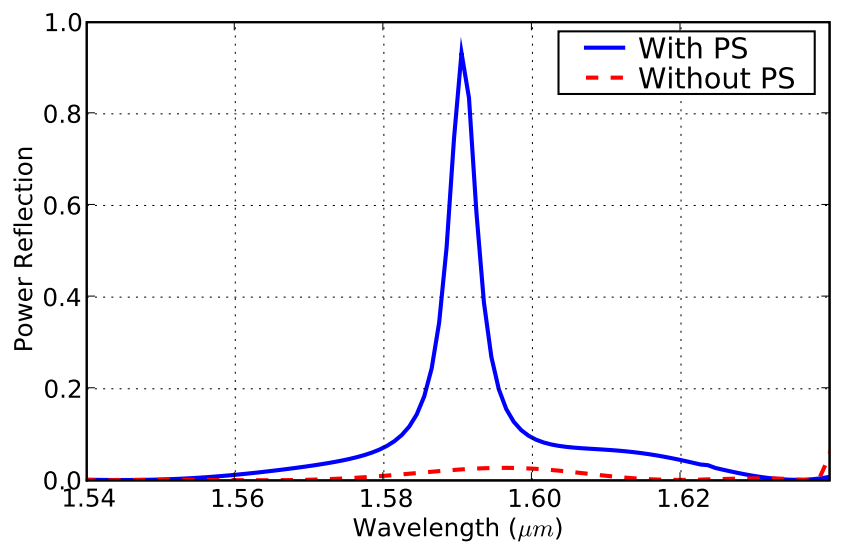

Fig. 2. Reflection spectra with and without a phase section (PS).

Figure 2 plots the spectra of the active waveguide to active waveguide reflection with and without a phase section. The dashed curve depicts the grating as an evanescent reflector in the Bragg regime. The limited overlap of the active waveguide mode with the silicon grating results in only $3 \%$ reflection. Adjusting the grating by adding a phase section, hence making it a resonant cavity, results in the solid curve, with a maximum reflection of $93 \%$.

Calculating the power profiles for both cases at the Bragg wavelength (which is where the reflection is maximum) illustrates the different reflection mechanisms. Figure 3 shows the optical power distributions in a cut similar to that of figure 1: the active waveguide is on top, the silicon grating on the bottom and ranges from $\mathrm{z}=1 \mu \mathrm{m}$ to $\mathrm{z}=19 \mu \mathrm{m}$. For clarity transparent rectangles indicate the location of both

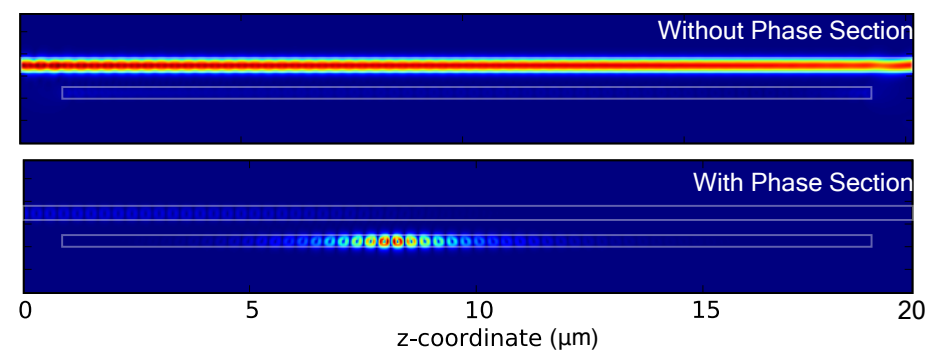

Fig. 3. Optical power profiles at Bragg wavelength $(\lambda=1.59 \mu \mathrm{m})$ with and without phase shifting section. Color scales differ for each plot.

waveguides. The first order mode of the active waveguide is excited from the left.

Without the phase section the grating acts as an evanescent Bragg reflector. In this case practically all the optical power is transmitted through the waveguide with a nearly uniform profile along the length of the active waveguide and negligible power is in the silicon waveguide. Near the far left side of the active waveguide some fluctuation in spatial power distribution can be observed due to the standing wave caused by the limited reflection.

The situation changes completely if a phase shifting section is added. In both waveguides a standing wave pattern is clearly visible due to the interference of forward and backward propagating waves. There is a very pronounced field enhancement in the silicon grating around the phase section (at approximately $\mathrm{z}=8 \mu \mathrm{m}$ ). The plot also shows how the light coupling from the cavity back into the active waveguide interferes destructively with the latter. Only $0.5 \%$ of the inbound power is transmitted to the far right side of the active waveguide while $\sim 93 \%$ of the power is reflected back into the laser cavity. The remaining $6.5 \%$ is lost, mainly due to scattering and some radiation at the edges of the silicon grating. Note that the color scales differ for each plot, but intensities can be compared by referring to the input power of the active waveguide.

\section{Conclusions}

We have presented a novel approach to design efficient hybrid microlasers by operating a silicon grating in a resonant state. A simulation example shows that this technique is capable of boosting the reflection from $3 \%$ to $93 \%$.

\section{REFERENCES}

[1] A. Fang et al. A distributed feedback silicon evanescent laser, Optics Express, Vol. 16, No 7, March 31, 2008

[2] T. Dupont et al. III-V on Si distributed-feedback lasers based on exchange Bragg contradirectionnal coupling, Proceedings of ECIO 2010, ThH2, Cambridge 2010

[3] J. Van Campenhout et al., A Compact SOI-Integrated Multiwavelength Laser Source Based on Cascaded InP Microdisks, IEEE Photonics Technology Letters, Vol. 20, No 16, August 15, 2008.

[4] CAMFR http://camfr.sourceforge.net 